\title{
Stroke Incidence, Rate of Thrombolytic Therapy, Mortality in Thailand from 2009 to 2021
}

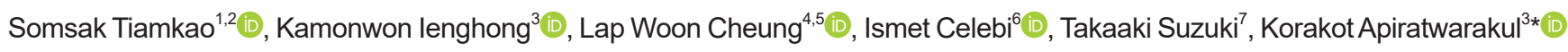 \\ ${ }^{1}$ Department of Medicine, Division of Neurology, Faculty of Medicine, Khon Kaen University, Khon Kaen, Thailand; ${ }^{2}$ Integrated \\ Epilepsy Research Group, Khon Kaen University, Khon Kaen, Thailand; ${ }^{3}$ Department of Emergency Medicine, Faculty of \\ Medicine, Khon Kaen University, Khon Kaen, Thailand; ${ }^{4}$ Department of Accident and Emergency, Princess Margaret Hospital, \\ Kowloon, Hong Kong; ${ }^{5}$ Emergency Medicine Unit, Li Ka Shing Faculty of Medicine, The University of Hong Kong, Pokfulam, \\ Hong Kong; ${ }^{6}$ Department of Paramedic, Gazi University, Ankara, Turkey; ${ }^{7}$ Department of Emergency and Critical Care Medicine, \\ University of Tsukuba Hospital, Tsukuba, Japan
}

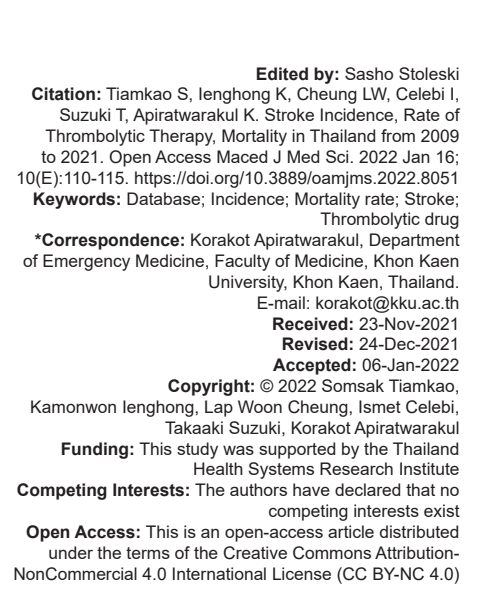

\section{Introduction}

Cerebrovascular disease, also known as stroke, is a common neurological condition that is the world's leading cause of death. According to a World Stroke Organization report Cerebrovascular disease is the leading cause of death in the world. Moreover, the World Health Organization second rank in terms of claiming the lives of world residents. Every year, around six million people die as a result of stroke and $10 \%$ of all forms of casualties are caused by this [1].

Stroke is the main cause of disability and death worldwide, with a high incidence rate. The death rate is high. It affects $16.0-23.0 \%$ of the world's population and is a major public health issue. Thailand has ranked stroke in the primary and secondary cause of death Thai women and Thai men, in that order. The death rate is high. The mortality rate is $10 \%$, with $50 \%$ of patients suffering from impairments. The Thai Ministry of Public
Health has a policy that encourages the development of new technologies. Stroke therapy recommendations patients should be prioritized to decrease costs as low as feasible the rate of mortality and disability [2].

Stroke is a major problem in the public health system in Thailand and around the world. Accordingly, stroke is the first cause of death and disability. The main treatment for acute ischemic stroke patient is thrombolytic agent therapy [3], including recombinant tissue plasminogen activator (rtPA). Thailand started rtPA treatment in 2008 only at university hospitals in Bangkok. After that, in 2009, began to treat in provincial university hospitals and gradually increased services in large provincial hospitals until widespread in 2014 with the support of the National Health Security Office (NHSO). At present, there are no data on the incidence of stroke patients, rtPA treatment rate, and mortality rate. However, no studies regard the services in stroke management in Thailand. This study aims to present data: incidence, rate of thrombolytic treatment, mortality rate of acute stroke in Thailand for 13 years. 


\section{Methods}

A study from the NHSO's database between 2009 and 2021 using percentage statistics, and the number of patients per 100,000 population. The database system is created by importing data from all hospitals in Thailand, both government and private sectors. Most of the respondents who have been trained to use the NHSO database are physicians or nurses working in stroke units. The NHSO database administrator will check the import of the data. If incomplete data are detected, it will be notified to the hospital where the data are imported to be corrected.

All data were gathered from NHSO database. The missing or incomplete data was excluded from this study. Double data entry protocol was used with Microsoft Excel 2019 (Khon Kaen University license). The data were interpreted through percentiles, means, standard deviations, and quartile ranges. The IBM SPSS statistics version 28 (Khon Kaen University license) was the tool for analyze this data.

Thailand is administratively divided into 76 provinces and one special administrative area (Bangkok), each of which has a large government hospital serving as a referral center for patients from community hospitals in the provinces in which it is responsible. With regard to the management of stroke patients, because rtPA is expensive and the limitations of Computerized Tomography scans, it has led to the integration of provinces with similar areas as a "healthy area" to save resources for managing stroke patients and reduce the duration of access to rtPA.

Ethical approval was provided by the Khon Kaen University Ethics Committee for Human Research (HE631574). Requirement for informed consent was waived since confidentiality protection had been guaranteed, as participants were not identified by name, but by a unique study number.

\section{Results}

The period of this study was 2009-2021. All data were conducted from NHSO database (Thai national database) with official permission to access stoke data. The area of Thailand in stroke care was divided into 13 healthy areas.

The overall incidence of acute stroke patients aged 15 years and over per 100,000 population is shown in Tables 1. It was found that the trend of incidence has been increasing ever since 2009 to the present. In 2020 , the healthy area 3 was highest incidence of acute stroke with $392.67 / 100,000$ population.

Incidence of acute ischemic stroke patients aged 15 years and over per 100,000 population is shown in Table 2. In 2009, the incidence of acute ischemic stroke was $90.37 / 100,000$ population as well as increasing to $203.66 / 100,000$ population in 2021 .

The incidence of non-traumatic intracerebral hemorrhage patients aged 15 years and over per 100,000 population is shown in Table 3 . In 2009, the incidence of non-traumatic intracerebral hemorrhage patients was $56.14 / 100,000$ population as well as increasing to $84.39 / 100,000$ population in 2021 .

The percentage of patients with acute ischemic stroke receiving rtPA continued to increase as shown in Table 4. From 2009 to 2021, the trend of patient who receives the rtPA was increasing (0.18 to $7.39 \%)$.

Percentage of mortality rate of acute stroke patients details as shown in Table 5. The trend of mortality rate in 13 years of the study was decreased from 14.77 to $10.87 \%$.

From Table 6, the mortality rate of acute stroke patients at day 30 of diagnosis was $16.94 \%$ in 2021 . The trend was decreased from 2009 (24.58\%).

The mortality rate of acute ischemic stroke patients is shown in Table 7 . Overall mortality rate in Thailand was decreased from 8.05 to $7.30 \%$ in 2021 .

From Table 8, the mortality rate of acute ischemic stroke patients at day 30 after diagnosis of stroke was decrease trend (15.93 to $9.36 \%)$ in the period of the study.

The mortality rate of non-traumatic intracerebral hemorrhage patients is shown in Table 9. Overall mortality rate in Thailand was decreased from 42.72 to $37.74 \%$ in 2021.

Patients with acute ischemic stroke who were treated with rtPA, the mortality rate is shown in Table 10. The trend was decreased from 10.53 to $7.30 \%$.

\section{Discussion}

The incidence of acute strokes and acute ischemic strokes increased from 2009 to 2021, and patient mortality rates have declined from 2009 to 2021 , consistent with the study by Krishnamurthi et al. [4] and Feigin et al. [5]. But unlike the study by Katan and Luft [6], in developed countries, incidence and mortality in stroke patients have declined over the past 30 years. The results of this previous study [6] are consistent with those of Giroud et al. [7]. The incidence of disease can be reduced depending on the control of the underlying disease, risk factors of stroke, non-communicable disease, diabetes, hypertension, and dyslipidemia [8].

Treatment with rtPA, the main study, the National Institute of Neurological Disorders and Stroke, found significant benefit to patients. The modified 
Table 1: Incidence of acute stroke patients aged 15 years and over per 100,000 population

\begin{tabular}{|c|c|c|c|c|c|c|c|c|c|c|c|c|c|}
\hline & 2009 & 2010 & 2011 & 2012 & 2013 & 2014 & 2015 & 2016 & 2017 & 2018 & 2019 & 2020 & 2021 \\
\hline Healthy area 1 & 186.63 & 189.35 & 206.04 & 214.82 & 220.59 & 234.66 & 242.12 & 263.43 & 253.16 & 280.02 & 297.59 & 299.36 & 289.25 \\
\hline Healthy area 2 & 202.86 & 206.67 & 232.13 & 246.11 & 259.38 & 273.71 & 282.09 & 289.34 & 284.84 & 319.76 & 329.61 & 326.76 & 302.72 \\
\hline Healthy area 3 & 239.82 & 250.19 & 278.79 & 296.05 & 313.22 & 332.74 & 334.13 & 341.37 & 347.34 & 369.01 & 387.20 & 392.67 & 361.57 \\
\hline Healthy area 4 & 230.32 & 230.06 & 267.57 & 277.70 & 277.25 & 298.34 & 311.04 & 326.28 & 333.50 & 344.04 & 356.32 & 348.74 & 328.35 \\
\hline Healthy area 5 & 204.19 & 199.42 & 223.82 & 239.46 & 249.77 & 267.41 & 288.28 & 296.70 & 300.04 & 329.86 & 346.01 & 349.45 & 312.81 \\
\hline Healthy area 6 & 213.07 & 217.06 & 235.17 & 247.29 & 253.67 & 272.44 & 280.52 & 307.02 & 313.11 & 331.41 & 342.26 & 336.29 & 301.90 \\
\hline Healthy area 7 & 121.37 & 133.94 & 150.85 & 164.32 & 174.00 & 198.16 & 226.17 & 239.6 & 247.69 & 272.67 & 284.97 & 292.42 & 272.20 \\
\hline Healthy area 8 & 129.77 & 143.03 & 154.15 & 161.34 & 174.23 & 188.15 & 211.83 & 228.00 & 243.34 & 264.57 & 275.69 & 289.79 & 270.59 \\
\hline Healthy area 9 & 161.71 & 172.86 & 195.30 & 206.75 & 220.60 & 250.15 & 268.72 & 290.54 & 304.81 & 340.77 & 358.53 & 363.41 & 342.11 \\
\hline Healthy area 10 & 126.88 & 135.04 & 162.48 & 172.01 & 187.77 & 202.10 & 217.23 & 230.63 & 249.37 & 269.83 & 279.95 & 303.45 & 274.04 \\
\hline Healthy area 11 & 147.02 & 155.41 & 174.07 & 185.64 & 198.91 & 217.61 & 232.62 & 249.25 & 271.35 & 290.76 & 318.66 & 325.05 & 292.10 \\
\hline Healthy area 12 & 135.11 & 149.02 & 166.75 & 171.37 & 181.60 & 201.34 & 221.21 & 243.77 & 255.96 & 295.54 & 318.67 & 307.00 & 281.39 \\
\hline Healthy area 13 & 168.38 & 185.17 & 178.96 & 195.00 & 206.16 & 216.64 & 230.52 & 241.26 & 250.10 & 264.32 & 280.22 & 346.40 & 194.15 \\
\hline Overall in Thailand & 172.33 & 179.86 & 199.25 & 210.16 & 220.67 & 238.57 & 253.94 & 270.00 & 278.49 & 303.20 & 318.89 & 328.00 & 303.03 \\
\hline
\end{tabular}

Table 2: Incidence of acute ischemic stroke patients aged 15 years and over per 100,000 population

\begin{tabular}{|c|c|c|c|c|c|c|c|c|c|c|c|c|c|}
\hline & 2009 & 2010 & 2011 & 2012 & 2013 & 2014 & 2015 & 2016 & 2017 & 2018 & 2019 & 2020 & 2021 \\
\hline Healthy area 1 & 95.99 & 101.20 & 107.10 & 114.87 & 123.25 & 136.74 & 145.28 & 160.80 & 155.00 & 170.40 & 186.59 & 189.62 & 177.96 \\
\hline Healthy area 2 & 93.12 & 97.59 & 116.70 & 141.05 & 148.86 & 161.73 & 173.97 & 161.00 & 169.00 & 200.00 & 213.96 & 208.17 & 191.68 \\
\hline Healthy area 3 & 119.08 & 135.30 & 148.50 & 165.68 & 182.43 & 198.30 & 200.38 & 206.80 & 220.00 & 228.40 & 250.85 & 257.94 & 232.47 \\
\hline Healthy area 4 & 112.58 & 120.30 & 143.70 & 153.78 & 160.71 & 176.95 & 194.45 & 198.80 & 212.00 & 215.20 & 229.59 & 223.15 & 209.64 \\
\hline Healthy area 5 & 96.45 & 105.60 & 127.40 & 137.53 & 151.12 & 162.74 & 183.43 & 184.10 & 192.00 & 211.70 & 227.24 & 235.31 & 202.67 \\
\hline Healthy area 6 & 113.41 & 128.90 & 137.30 & 151.99 & 161.85 & 176.07 & 186.99 & 205.10 & 208.00 & 225.60 & 233.33 & 218.83 & 199.50 \\
\hline Healthy area 7 & 56.51 & 71.13 & 80.87 & 94.23 & 101.20 & 126.00 & 151.28 & 158.80 & 172.00 & 193.00 & 208.90 & 211.25 & 194.41 \\
\hline Healthy area 8 & 68.90 & 79.68 & 85.96 & 85.63 & 106.51 & 119.98 & 140.84 & 155.80 & 170.00 & 186.40 & 201.36 & 208.79 & 196.68 \\
\hline Healthy area 9 & 77.48 & 95.07 & 108.10 & 118.61 & 131.43 & 157.92 & 172.92 & 194.20 & 215.00 & 244.80 & 253.65 & 258.18 & 237.96 \\
\hline Healthy area 10 & 70.77 & 80.78 & 93.77 & 103.52 & 113.14 & 123.33 & 135.70 & 144.10 & 173.00 & 186.40 & 197.28 & 209.22 & 182.77 \\
\hline Healthy area 11 & 78.03 & 89.46 & 104.70 & 110.51 & 119.85 & 129.56 & 141.15 & 155.20 & 173.00 & 183.60 & 211.93 & 218.87 & 197.37 \\
\hline Healthy area 12 & 67.86 & 79.27 & 92.17 & 101.57 & 111.55 & 124.61 & 137.84 & 158.80 & 179.00 & 207.30 & 233.49 & 220.47 & 201.57 \\
\hline Healthy area 13 & 91.39 & 107.30 & 101.30 & 113.09 & 122.96 & 137.45 & 141.48 & 145.30 & 164.00 & 175.40 & 188.26 & 235.91 & 133.88 \\
\hline Overall in Thailand & 90.37 & 100.80 & 112.60 & 122.78 & 134.03 & 146.61 & 160.36 & 170.90 & 184.00 & 202.00 & 217.58 & 222.73 & 203.66 \\
\hline
\end{tabular}

Table 3: Incidence of non-traumatic intracerebral hemorrhage patients aged 15 years and over per 100,000 population

\begin{tabular}{|c|c|c|c|c|c|c|c|c|c|c|c|c|c|}
\hline & 2009 & 2010 & 2011 & 2012 & 2013 & 2014 & 2015 & 2016 & 2017 & 2018 & 2019 & 2020 & 2021 \\
\hline Healthy area 1 & 62.74 & 61.65 & 72.14 & 69.34 & 68.69 & 73.35 & 78.71 & 79.84 & 81.82 & 89.22 & 88.11 & 89.42 & 90.97 \\
\hline Healthy area 2 & 71.65 & 70.03 & 81.88 & 79.59 & 83.39 & 88.17 & 87.85 & 93.64 & 94.60 & 103.36 & 101.14 & 101.79 & 98.00 \\
\hline Healthy area 3 & 81.53 & 80.70 & 97.08 & 94.23 & 95.19 & 98.76 & 97.54 & 97.69 & 105.76 & 114.31 & 112.99 & 112.71 & 110.40 \\
\hline Healthy area 4 & 82.01 & 75.04 & 90.53 & 94.06 & 85.75 & 92.90 & 89.10 & 94.07 & 102.25 & 104.90 & 101.23 & 100.65 & 95.32 \\
\hline Healthy area 5 & 68.64 & 64.52 & 71.69 & 76.97 & 76.27 & 81.81 & 83.01 & 84.41 & 88.87 & 95.58 & 96.71 & 97.89 & 93.11 \\
\hline Healthy area 6 & 70.28 & 68.86 & 73.47 & 78.02 & 76.21 & 82.78 & 80.74 & 86.12 & 93.45 & 93.53 & 92.65 & 96.13 & 85.46 \\
\hline Healthy area 7 & 35.99 & 38.71 & 46.71 & 49.08 & 45.50 & 51.43 & 57.42 & 59.84 & 62.29 & 69.86 & 66.65 & 72.40 & 70.88 \\
\hline Healthy area 8 & 33.77 & 36.97 & 42.66 & 41.84 & 41.61 & 48.90 & 51.03 & 51.95 & 57.40 & 56.46 & 59.42 & 64.45 & 64.14 \\
\hline Healthy area 9 & 54.78 & 55.16 & 67.43 & 64.84 & 65.61 & 70.56 & 74.47 & 77.11 & 81.82 & 85.53 & 86.66 & 91.78 & 92.23 \\
\hline Healthy area 10 & 39.02 & 40.43 & 54.31 & 51.12 & 53.74 & 57.48 & 60.03 & 63.04 & 68.88 & 74.24 & 73.95 & 77.89 & 78.06 \\
\hline Healthy area 11 & 41.42 & 43.17 & 48.24 & 49.84 & 52.52 & 60.95 & 60.43 & 64.95 & 69.72 & 71.97 & 74.05 & 76.31 & 71.76 \\
\hline Healthy area 12 & 41.67 & 42.26 & 47.70 & 46.24 & 46.95 & 50.61 & 55.72 & 58.03 & 64.82 & 71.85 & 70.84 & 73.21 & 65.29 \\
\hline Healthy area 13 & 55.30 & 56.84 & 58.33 & 58.57 & 63.32 & 62.67 & 65.88 & 70.16 & 75.41 & 76.60 & 76.41 & 96.65 & 54.52 \\
\hline Overall in Thailand & 56.14 & 55.77 & 64.68 & 64.65 & 64.58 & 69.43 & 71.33 & 74.23 & 79.27 & 83.57 & 83.24 & 87.63 & 84.39 \\
\hline
\end{tabular}

Table 4: Percentage of acute ischemic stroke patients who treated with rtPA

\begin{tabular}{|c|c|c|c|c|c|c|c|c|c|c|c|c|c|}
\hline & 2009 & 2010 & 2011 & 2012 & 2013 & 2014 & 2015 & 2016 & 2017 & 2018 & 2019 & 2020 & 2021 \\
\hline Healthy area 1 & 0.09 & 1.61 & 3.08 & 3.24 & 5.04 & 5.47 & 5.15 & 6.41 & 7.65 & 7.95 & 8.66 & 8.50 & 8.43 \\
\hline Healthy area 2 & 0.00 & 0.21 & 1.54 & 2.31 & 4.41 & 7.73 & 6.79 & 6.88 & 7.31 & 9.06 & 10.22 & 10.70 & 9.70 \\
\hline Healthy area 3 & 0.05 & 0.00 & 0.07 & 0.30 & 1.05 & 2.91 & 3.78 & 3.19 & 4.78 & 7.61 & 9.05 & 8.79 & 6.53 \\
\hline Healthy area 4 & 0.34 & 2.00 & 5.19 & 5.44 & 5.23 & 5.82 & 5.26 & 5.46 & 5.00 & 5.36 & 7.01 & 6.41 & 6.10 \\
\hline Healthy area 5 & 0.04 & 0.00 & 0.00 & 0.43 & 0.98 & 1.97 & 2.01 & 3.56 & 4.87 & 6.21 & 7.01 & 7.47 & 6.52 \\
\hline Healthy area 6 & 0.36 & 5.17 & 1.51 & 1.65 & 2.15 & 2.83 & 3.47 & 3.30 & 4.29 & 5.04 & 7.34 & 6.97 & 6.52 \\
\hline Healthy area 7 & 0.00 & 1.94 & 2.77 & 4.18 & 4.30 & 4.79 & 5.98 & 5.98 & 7.94 & 8.17 & 8.08 & 10.26 & 9.69 \\
\hline Healthy area 8 & 0.00 & 0.00 & 0.49 & 2.82 & 5.30 & 5.04 & 6.55 & 5.41 & 5.55 & 6.75 & 7.11 & 6.34 & 5.77 \\
\hline Healthy area 9 & 0.00 & 0.25 & 1.04 & 1.86 & 2.14 & 2.27 & 2.71 & 3.73 & 4.21 & 4.64 & 6.02 & 6.15 & 5.87 \\
\hline Healthy area 10 & 0.00 & 0.49 & 0.50 & 0.17 & 0.41 & 2.12 & 3.29 & 4.64 & 7.69 & 7.67 & 9.22 & 9.58 & 8.60 \\
\hline Healthy area 11 & 0.19 & 1.13 & 1.70 & 2.49 & 2.40 & 3.50 & 3.55 & 4.27 & 4.31 & 7.19 & 10.12 & 10.78 & 10.25 \\
\hline Healthy area 12 & 0.25 & 0.35 & 0.95 & 1.50 & 3.20 & 3.51 & 4.33 & 5.25 & 6.44 & 5.89 & 6.55 & 7.45 & 8.29 \\
\hline Healthy area 13 & 0.69 & 1.17 & 1.62 & 1.90 & 2.62 & 3.15 & 3.51 & 5.28 & 6.33 & 6.80 & 6.93 & 7.77 & 6.07 \\
\hline Overall in Thailand & 0.18 & 1.24 & 1.62 & 2.19 & 3.02 & 3.85 & 4.26 & 4.82 & 5.77 & 6.59 & 7.75 & 8.04 & 7.39 \\
\hline
\end{tabular}

Table 5: Percentage of mortality rate of acute stroke patients

\begin{tabular}{|c|c|c|c|c|c|c|c|c|c|c|c|c|c|}
\hline & 2009 & 2010 & 2011 & 2012 & 2013 & 2014 & 2015 & 2016 & 2017 & 2018 & 2019 & 2020 & 2021 \\
\hline Healthy area 1 & 10.10 & 11.37 & 11.29 & 10.44 & 10.34 & 9.02 & 9.81 & 8.77 & 8.39 & 8.36 & 8.56 & 8.84 & 9.63 \\
\hline Healthy area 2 & 15.88 & 17.20 & 16.07 & 14.35 & 14.04 & 13.82 & 14.27 & 13.99 & 13.50 & 13.55 & 11.62 & 12.14 & 13.22 \\
\hline Healthy area 3 & 18.85 & 17.87 & 17.74 & 17.54 & 17.97 & 16.12 & 16.93 & 15.55 & 13.67 & 15.64 & 14.36 & 13.71 & 14.82 \\
\hline Healthy area 4 & 22.11 & 22.79 & 21.60 & 20.59 & 19.81 & 18.41 & 17.31 & 17.93 & 16.00 & 16.20 & 15.02 & 15.36 & 16.80 \\
\hline Healthy area 5 & 17.49 & 17.07 & 16.08 & 17.28 & 16.20 & 15.39 & 14.09 & 13.62 & 13.77 & 13.16 & 12.41 & 12.90 & 13.47 \\
\hline Healthy area 6 & 20.54 & 20.19 & 18.58 & 19.30 & 18.41 & 18.43 & 16.63 & 15.86 & 15.51 & 14.18 & 14.04 & 14.22 & 14.75 \\
\hline Healthy area 7 & 7.88 & 7.59 & 7.16 & 7.03 & 6.00 & 6.78 & 5.87 & 5.08 & 5.35 & 5.10 & 5.01 & 4.86 & 5.23 \\
\hline Healthy area 8 & 5.06 & 5.56 & 5.81 & 5.99 & 5.39 & 5.71 & 6.12 & 5.18 & 4.85 & 4.19 & 4.60 & 4.56 & 5.25 \\
\hline Healthy area 9 & 14.17 & 14.87 & 14.66 & 14.35 & 13.70 & 12.36 & 11.46 & 11.03 & 9.97 & 8.95 & 8.75 & 9.47 & 9.40 \\
\hline Healthy area 10 & 8.59 & 9.06 & 8.60 & 8.25 & 8.86 & 7.81 & 7.26 & 6.46 & 6.49 & 6.75 & 7.00 & 7.31 & 7.75 \\
\hline Healthy area 11 & 14.76 & 14.32 & 14.70 & 13.62 & 13.56 & 12.92 & 12.23 & 13.11 & 11.36 & 10.93 & 10.61 & 10.27 & 10.86 \\
\hline Healthy area 12 & 10.56 & 9.80 & 9.37 & 10.76 & 10.07 & 8.62 & 9.41 & 9.15 & 8.60 & 7.16 & 7.38 & 7.44 & 6.99 \\
\hline Healthy area 13 & 17.03 & 15.83 & 15.21 & 15.29 & 14.42 & 13.58 & 13.48 & 13.27 & 12.57 & 12.58 & 11.99 & 12.02 & 12.87 \\
\hline Overall in Thailand & 14.77 & 14.73 & 14.20 & 14.06 & 13.49 & 12.69 & 12.22 & 11.75 & 11.02 & 10.60 & 10.24 & 10.35 & 10.87 \\
\hline
\end{tabular}


Table 6: Percentage of mortality rate of acute stroke patients at day $\mathbf{3 0}$

\begin{tabular}{|c|c|c|c|c|c|c|c|c|c|c|c|c|c|}
\hline & 2009 & 2010 & 2011 & 2012 & 2013 & 2014 & 2015 & 2016 & 2017 & 2018 & 2019 & 2020 & 2021 \\
\hline Healthy area 1 & 24.59 & 25.02 & 24.08 & 22.19 & 22.16 & 20.9 & 21.07 & 19.97 & 18.09 & 17.87 & 17.14 & 17.42 & 18.11 \\
\hline Healthy area 2 & 25.87 & 26.47 & 24.18 & 22.40 & 21.76 & 21.77 & 19.97 & 20.42 & 19.61 & 19.35 & 16.82 & 17.79 & 18.53 \\
\hline Healthy area 3 & 27.75 & 26.26 & 27.39 & 25.56 & 24.77 & 22.74 & 23.19 & 21.03 & 18.97 & 20.20 & 19.13 & 18.60 & 20.07 \\
\hline Healthy area 4 & 24.32 & 26.03 & 23.70 & 22.63 & 22.15 & 20.22 & 18.89 & 19.77 & 17.98 & 18.26 & 16.48 & 17.51 & 17.98 \\
\hline Healthy area 5 & 23.53 & 22.66 & 21.66 & 21.81 & 20.02 & 19.02 & 17.65 & 17.01 & 16.99 & 16.13 & 14.78 & 15.83 & 17.15 \\
\hline Healthy area 6 & 23.61 & 22.89 & 21.71 & 21.63 & 20.50 & 20.39 & 19.44 & 18.67 & 18.27 & 16.86 & 16.11 & 17.02 & 17.23 \\
\hline Healthy area 7 & 30.40 & 28.78 & 28.93 & 26.47 & 25.12 & 24.13 & 22.45 & 20.44 & 19.72 & 17.92 & 16.36 & 17.05 & 17.80 \\
\hline Healthy area 8 & 23.24 & 22.34 & 22.95 & 20.70 & 19.54 & 19.76 & 18.80 & 18.15 & 15.67 & 14.06 & 14.19 & 14.59 & 15.59 \\
\hline Healthy area 9 & 28.43 & 29.06 & 27.92 & 25.49 & 25.37 & 22.85 & 21.64 & 20.61 & 18.08 & 17.18 & 16.05 & 16.89 & 17.25 \\
\hline Healthy area 10 & 26.65 & 26.11 & 25.94 & 24.74 & 23.56 & 22.15 & 20.83 & 19.12 & 18.22 & 18.09 & 16.43 & 15.97 & 17.06 \\
\hline Healthy area 11 & 22.96 & 23.58 & 22.36 & 20.44 & 20.85 & 19.54 & 18.58 & 18.45 & 16.34 & 15.84 & 15.25 & 14.70 & 15.16 \\
\hline Healthy area 12 & 21.35 & 22.65 & 21.03 & 20.72 & 19.69 & 17.91 & 18.44 & 17.25 & 16.75 & 15.33 & 14.50 & 14.00 & 14.04 \\
\hline Healthy area 13 & 17.59 & 16.04 & 15.62 & 15.95 & 14.95 & 14.41 & 14.00 & 14.25 & 13.49 & 13.24 & 12.93 & 13.21 & 14.12 \\
\hline Overall in Thailand & 24.58 & 24.38 & 23.66 & 22.43 & 21.63 & 20.48 & 19.61 & 18.88 & 17.56 & 16.89 & 15.84 & 16.21 & 16.94 \\
\hline
\end{tabular}

Table 7: Percentage of mortality rate of acute ischemic stroke patients

\begin{tabular}{|c|c|c|c|c|c|c|c|c|c|c|c|c|c|}
\hline & 2009 & 2010 & 2011 & 2012 & 2013 & 2014 & 2015 & 2016 & 2017 & 2018 & 2019 & 2020 & 2021 \\
\hline Healthy area 1 & 5.25 & 6.46 & 5.46 & 5.21 & 5.26 & 5.05 & 5.03 & 4.24 & 4.96 & 5.51 & 5.39 & 7.23 & 8.24 \\
\hline Healthy area 2 & 8.51 & 9.08 & 7.49 & 7.44 & 6.03 & 6.54 & 7.20 & 6.97 & 6.53 & 7.74 & 5.62 & 7.41 & 9.55 \\
\hline Healthy area 3 & 10.23 & 11.24 & 9.89 & 8.87 & 10.34 & 7.94 & 8.22 & 7.89 & 8.74 & 9.86 & 9.35 & 8.23 & 10.42 \\
\hline Healthy area 4 & 12.69 & 12.84 & 11.06 & 10.95 & 10.69 & 8.34 & 7.91 & 8.91 & 6.39 & 7.29 & 9.03 & 7.43 & 10.74 \\
\hline Healthy area 5 & 9.10 & 9.46 & 8.80 & 9.00 & 8.25 & 7.71 & 6.75 & 6.07 & 7.12 & 10.94 & 6.74 & 10.15 & 8.67 \\
\hline Healthy area 6 & 11.02 & 10.76 & 9.57 & 9.72 & 9.03 & 9.23 & 7.82 & 7.39 & 11.76 & 6.61 & 7.65 & 9.94 & 7.19 \\
\hline Healthy area 7 & 4.70 & 3.92 & 3.60 & 3.78 & 3.49 & 3.54 & 3.16 & 2.27 & 2.46 & 3.65 & 2.59 & 2.70 & 2.38 \\
\hline Healthy area 8 & 2.68 & 3.48 & 3.57 & 4.48 & 2.66 & 2.99 & 3.03 & 2.74 & 2.57 & 1.47 & 3.16 & 3.83 & 4.30 \\
\hline Healthy area 9 & 8.05 & 8.79 & 7.37 & 6.89 & 7.53 & 6.45 & 5.71 & 5.14 & 6.99 & 7.25 & 5.91 & 7.01 & 6.55 \\
\hline Healthy area 10 & 5.23 & 6.00 & 4.35 & 4.82 & 5.64 & 4.81 & 4.03 & 3.39 & 2.84 & 3.69 & 3.12 & 4.63 & 6.10 \\
\hline Healthy area 11 & 8.98 & 8.60 & 8.29 & 6.68 & 7.60 & 6.87 & 6.49 & 7.21 & 8.96 & 6.18 & 6.37 & 9.16 & 8.90 \\
\hline Healthy area 12 & 5.51 & 6.09 & 5.27 & 5.64 & 5.99 & 4.70 & 4.98 & 5.08 & 9.30 & 5.88 & 6.62 & 6.53 & 7.77 \\
\hline Healthy area 13 & 9.48 & 8.54 & 7.98 & 8.21 & 8.22 & 7.14 & 7.96 & 7.53 & 6.49 & 8.54 & 6.07 & 8.23 & 6.33 \\
\hline Overall in Thailand & 8.05 & 8.33 & 7.37 & 7.31 & 7.19 & 6.44 & 6.09 & 5.78 & 6.24 & 6.38 & 5.94 & 7.07 & 7.30 \\
\hline
\end{tabular}

Table 8: Percentage of mortality rate of acute ischemic stroke patients at day $\mathbf{3 0}$

\begin{tabular}{|c|c|c|c|c|c|c|c|c|c|c|c|c|c|}
\hline & 2009 & 2010 & 2011 & 2012 & 2013 & 2014 & 2015 & 2016 & 2017 & 2018 & 2019 & 2020 & 2021 \\
\hline Healthy area 1 & 16.09 & 17.09 & 15.45 & 14.56 & 15.02 & 14.25 & 13.20 & 13.17 & 10.55 & 10.62 & 10.96 & 10.55 & 11.01 \\
\hline Healthy area 2 & 16.44 & 15.82 & 13.60 & 13.69 & 11.80 & 12.18 & 11.61 & 12.00 & 11.26 & 10.90 & 8.74 & 9.52 & 9.52 \\
\hline Healthy area 3 & 17.90 & 18.45 & 16.63 & 15.71 & 15.87 & 14.07 & 13.41 & 12.89 & 10.82 & 10.93 & 11.47 & 11.12 & 11.07 \\
\hline Healthy area 4 & 15.51 & 16.64 & 12.95 & 13.07 & 13.95 & 10.85 & 10.38 & 11.25 & 9.69 & 9.96 & 8.75 & 9.33 & 9.40 \\
\hline Healthy area 5 & 14.04 & 14.18 & 13.80 & 12.97 & 11.86 & 10.80 & 10.08 & 9.38 & 9.52 & 9.11 & 7.35 & 8.35 & 9.16 \\
\hline Healthy area 6 & 14.07 & 14.15 & 12.90 & 12.17 & 11.61 & 11.44 & 10.58 & 9.93 & 9.74 & 8.93 & 8.85 & 9.24 & 9.22 \\
\hline Healthy area 7 & 23.62 & 19.96 & 19.71 & 17.84 & 17.97 & 16.41 & 14.68 & 13.28 & 12.78 & 11.10 & 9.67 & 10.26 & 10.78 \\
\hline Healthy area 8 & 17.11 & 16.25 & 16.18 & 14.64 & 13.03 & 12.49 & 12.09 & 11.49 & 9.01 & 8.18 & 8.58 & 8.64 & 8.43 \\
\hline Healthy area 9 & 18.87 & 19.62 & 16.84 & 14.99 & 16.51 & 14.69 & 13.57 & 12.67 & 9.85 & 9.20 & 8.49 & 9.67 & 9.00 \\
\hline Healthy area 10 & 19.57 & 19.64 & 17.62 & 17.65 & 16.26 & 16.19 & 13.77 & 11.95 & 10.6 & 10.35 & 8.61 & 9.16 & 9.40 \\
\hline Healthy area 11 & 15.07 & 15.27 & 13.60 & 12.06 & 13.41 & 12.10 & 11.45 & 10.98 & 9.04 & 9.15 & 9.00 & 8.93 & 8.32 \\
\hline Healthy area 12 & 12.39 & 14.53 & 13.00 & 12.39 & 12.42 & 11.02 & 11.13 & 10.43 & 9.41 & 8.84 & 8.48 & 7.87 & 8.46 \\
\hline Healthy area 13 & 10.21 & 8.74 & 8.96 & 9.56 & 9.16 & 8.20 & 8.68 & 8.51 & 8.05 & 7.05 & 7.82 & 8.07 & 8.76 \\
\hline Overall in Thailand & 15.93 & 15.98 & 14.6 & 13.84 & 13.70 & 12.60 & 11.85 & 11.32 & 9.96 & 9.45 & 8.90 & 9.25 & 9.36 \\
\hline
\end{tabular}

Table 9: Percentage of mortality rate of non-traumatic intracerebral hemorrhage patients at day $\mathbf{3 0}$

\begin{tabular}{|c|c|c|c|c|c|c|c|c|c|c|c|c|c|}
\hline & 2009 & 2010 & 2011 & 2012 & 2013 & 2014 & 2015 & 2016 & 2017 & 2018 & 2019 & 2020 & 2021 \\
\hline Healthy area 1 & 39.97 & 40.71 & 39.88 & 37.85 & 37.96 & 35.57 & 37.25 & 36.00 & 35.99 & 35.28 & 33.10 & 35.21 & 35.02 \\
\hline Healthy area 2 & 42.34 & 46.57 & 42.29 & 41.37 & 42.70 & 41.46 & 38.54 & 39.96 & 37.99 & 38.84 & 36.24 & 36.66 & 38.12 \\
\hline Healthy area 3 & 47.47 & 45.53 & 49.52 & 48.89 & 46.30 & 45.04 & 48.78 & 44.25 & 39.73 & 43.98 & 41.12 & 39.69 & 42.25 \\
\hline Healthy area 4 & 41.49 & 45.46 & 43.92 & 41.93 & 41.64 & 41.76 & 40.36 & 41.52 & 38.37 & 38.82 & 37.87 & 38.49 & 39.45 \\
\hline Healthy area 5 & 42.05 & 41.13 & 39.06 & 40.13 & 39.06 & 37.80 & 36.57 & 36.23 & 35.92 & 34.58 & 34.64 & 36.48 & 36.91 \\
\hline Healthy area 6 & 42.45 & 41.71 & 41.27 & 41.16 & 40.57 & 40.81 & 40.66 & 40.18 & 38.99 & 38.71 & 36.75 & 37.42 & 38.02 \\
\hline Healthy area 7 & 47.65 & 50.45 & 49.33 & 46.15 & 46.33 & 46.82 & 43.58 & 41.92 & 41.83 & 37.83 & 38.38 & 39.17 & 38.31 \\
\hline Healthy area 8 & 40.31 & 39.38 & 41.28 & 39.78 & 40.97 & 40.42 & 41.77 & 41.15 & 38.69 & 36.99 & 37.14 & 36.68 & 39.69 \\
\hline Healthy area 9 & 48.89 & 49.06 & 48.97 & 48.62 & 48.52 & 45.45 & 43.62 & 43.14 & 41.78 & 41.94 & 40.09 & 39.81 & 39.92 \\
\hline Healthy area 10 & 44.51 & 42.33 & 42.96 & 43.17 & 43.49 & 40.93 & 41.90 & 40.06 & 40.50 & 41.80 & 39.10 & 38.32 & 38.82 \\
\hline Healthy area 11 & 45.40 & 45.14 & 45.09 & 44.20 & 42.75 & 40.42 & 40.59 & 40.48 & 39.09 & 37.71 & 37.13 & 36.03 & 37.73 \\
\hline Healthy area 12 & 39.73 & 43.50 & 41.12 & 41.83 & 39.33 & 37.87 & 40.05 & 38.00 & 38.54 & 35.80 & 36.07 & 34.07 & 33.25 \\
\hline Healthy area 13 & 32.16 & 31.97 & 28.52 & 30.32 & 28.09 & 28.90 & 27.72 & 28.63 & 27.28 & 28.54 & 27.06 & 26.67 & 28.33 \\
\hline Overall in Thailand & 42.72 & 43.35 & 42.69 & 42.15 & 41.45 & 40.38 & 40.11 & 39.47 & 38.23 & 38.03 & 36.74 & 36.84 & 37.74 \\
\hline
\end{tabular}

Table 10: Percentage of mortality rate of patients who treated with rtPA

\begin{tabular}{|c|c|c|c|c|c|c|c|c|c|c|c|c|c|}
\hline & 2009 & 2010 & 2011 & 2012 & 2013 & 2014 & 2015 & 2016 & 2017 & 2018 & 2019 & 2020 & 2021 \\
\hline Healthy area 1 & 33.33 & 5.26 & 8.77 & 3.05 & 4.98 & 6.15 & 5.47 & 3.74 & 4.96 & 5.51 & 5.39 & 7.23 & 8.24 \\
\hline Healthy area 2 & NA & NA & 11.11 & 5.88 & 5.76 & 8.84 & 8.05 & 7.66 & 6.53 & 7.74 & 5.62 & 7.41 & 9.55 \\
\hline Healthy area 3 & NA & NA & 50.00 & NA & 8.57 & 8.91 & 1.52 & 7.83 & 8.74 & 9.86 & 9.35 & 8.23 & 10.42 \\
\hline Healthy area 4 & NA & 6.78 & 4.35 & 5.66 & 5.17 & 2.72 & 6.48 & 6.83 & 6.39 & 7.29 & 9.03 & 7.43 & 10.74 \\
\hline Healthy area 5 & NA & NA & NA & 11.11 & 13.33 & 6.32 & 6.48 & 8.59 & 7.12 & 10.94 & 6.74 & 10.15 & 8.67 \\
\hline Healthy area 6 & 16.67 & 2.07 & 12.70 & 15.19 & 13.51 & 9.21 & 10.38 & 7.27 & 11.76 & 6.61 & 7.65 & 9.94 & 7.19 \\
\hline Healthy area 7 & NA & 2.38 & 2.86 & 3.23 & 1.44 & 3.89 & 3.31 & 2.11 & 2.46 & 3.65 & 2.59 & 2.70 & 2.38 \\
\hline Healthy area 8 & NA & NA & NA & 10.00 & 4.12 & 4.55 & 3.33 & 4.69 & 2.57 & 1.47 & 3.16 & 3.83 & 4.30 \\
\hline Healthy area 9 & NA & 11.11 & 2.33 & 13.95 & 17.27 & 10.37 & 6.86 & 6.55 & 6.99 & 7.25 & 5.91 & 7.01 & 6.55 \\
\hline Healthy area 10 & NA & NA & 7.69 & NA & NA & 5.71 & 2.50 & 1.69 & 2.84 & 3.69 & 3.12 & 4.63 & 6.10 \\
\hline Healthy area 11 & NA & 3.70 & 10.42 & 2.67 & 8.97 & 9.84 & 10.37 & 8.47 & 8.96 & 6.18 & 6.37 & 9.16 & 8.90 \\
\hline Healthy area 12 & NA & NA & 7.69 & 6.52 & 8.11 & 5.22 & 5.43 & 6.25 & 9.30 & 5.88 & 6.62 & 6.53 & 7.77 \\
\hline Healthy area 13 & 13.64 & 9.30 & 5.00 & 7.69 & 9.16 & 6.58 & 8.29 & 5.48 & 6.49 & 8.54 & 6.07 & 8.23 & 6.33 \\
\hline Overall in Thailand & 10.53 & 3.97 & 6.70 & 6.82 & 7.18 & 6.51 & 6.00 & 5.73 & 6.24 & 6.38 & 5.94 & 7.07 & 7.30 \\
\hline
\end{tabular}

rtPA: Recombinant tissue plasminogen activator, NA: Not applicable 
Rankin Scale 0-1 at 3 months was $42.6 \%$ in the rtPAtreated group, $26.6 \%$ in the non rtPA-treated group, $p<0.01$, and the number needed to treat to benefit equals 6 . Thrombolytic treatment with rtPA is the standard treatment for patients with acute ischemic stroke, and the National Health Security Administration supports this standard therapy. All patients do not pay for treatment at all, which is estimated at 2000 US dollars per treatment. The limitation of treatment is neurologist available and must be treated within $270 \mathrm{~min}$ of neurological symptoms. Therefore, this treatment system has been developed by creating a network of internists and emergency physicians under the consultation of a neurologist; this treatment has been extended to all provinces in Thailand [9]. And also has been designated as a service plan for stroke treatment as one of the health system services of the Ministry of Health. There must be continuous monitoring of the performance from the management. This led to an increase in the number of patients with acute ischemic stroke treated with rtPA from $0.18 \%$ in 2009 to $8.04 \%$ in 2020 .

Outcomes of patients with acute ischemic stroke treated with rtPA by internist or an emergency physician under the guidance of a neurologist, the results were not different from the neurologist [9], [10]. Therefore, the mortality rate for patients with acute ischemic stroke treated with rtPA in each health area was similar as shown in Table 10. And also, the mortality rate was not different from all patients with acute ischemic stroke as shown in Table 7 . The treatment with rtPA was found that the faster treatment, the better outcome. It has a better effect. Patient mortality at 1 year was associated with an increase in door to needle every 15 min [11]. Hospitals treating patients with acute ischemic stroke with rtPA must pass hospital accreditation. The treatment results to be better [12]. It was also found that if the patient had the number of microbleed lesions of more than 10 sites on the MRI brain, the mortality rate was higher [13].

Factors affecting the mortality of patients with acute stroke in Thailand were complications during treatment such as aspiration pneumonia [14], sepsis, infective endocarditis [15] and also, co-morbidities such as diabetes [16], coronary heart disease [17], chronic kidney disease [18], HIV infection [19], and rheumatic heart disease [20]. The mortality rate for patients with acute ischemic stroke at 30 days is likely to decline from $15.98 \%$ in 2010 to 8.9 in 2019 , consistent with the di Poggio et al. [21].

Patients with non-traumatic intracerebral hemorrhage had a significantly higher mortality rate in the first 30 days than those with acute ischemic stroke. From 2009 to 2021, the mortality rate ranged from $36.74 \%$ to $43.35 \%$. This tends to decrease somewhat but not as much as the mortality rate of acute ischemic stroke, which is consistent with the study by Pinho et al. [22] found a mortality rate of approximately $40 \%$. Treatment outcomes and patient mortality rates non-traumatic intracerebral hemorrhage can be reduced by urgent correction of bleeding tendency and the goal of high blood pressure is controlled [23].

The limitation of this study was that individual patient details were not available. Therefore, it is not possible to determine the severity of the disease, co-morbidity. The highlight of this study is a 12-year study that started rtPA therapy until now. This led to a trend in outcomes of treatments up to 12 years. Further studies that should be added to this study include enrollment of all stroke patients for further study in detail.

\section{Conclusions}

Stroke incidence tends to increase, with acute ischemic stroke patients receiving thrombolytic treatment tend to increase, too. The mortality rate in patients with acute stroke tends to decline. Especially in patients with acute ischemic stroke, the mortality rate was greatly reduced.

\section{References}

1. Tiamkao S, Pearkao C, Yubolchit N. Can stroke fast track improve quality of life of patients with acute ischemic stroke? J Med Assoc Thai. 2020;103 Suppl 6:35-41.

2. Phongphuttha $\mathrm{W}$, Tiamkao $\mathrm{S}$. Outcome of stroke fast-track patients arrival by emergency medical services. J Med Assoc Thai. 2021;104 Suppl 1:S88-93. https://doi.org/10.35755/ jmedassocthai.2021.S01.12299

3. Prabhakaran S, Ruff I, Bernstein RA. Acute stroke intervention: A systematic review. JAMA. 2015;313(14):1451-62. https://doi. org/10.1001/jama.2015.3058 PMid:25871671

4. Krishnamurthi RV, Moran AE, Feigin VL, Barker-Collo $\mathrm{S}$, Norrving B, Mensah GA, et al. Stroke prevalence, mortality and disability-adjusted life years in adults aged 20-64 years in 1990-2013: Data from the global burden of disease 2013 Study. Neuroepidemiology. 2015;45(3):190-202. https://doi. org/10.1159/000441098

PMid:26505983

5. Feigin VL, Forouzanfar $\mathrm{MH}$, Krishnamurthi R, Mensah GA Connor M, Bennett DA, et al. Global and regional burden of stroke during 1990-2010: Findings from the global burden of disease study 2010. Lancet. 2014;383(9913):245-54. https:// doi.org/10.1016/s0140-6736(13)61953-4 PMid:24449944

6. Katan M, Luft A. Global burden of stroke. Semin Neurol. 2018;38(2):208-11. https://doi.org/10.1055/s-0038-1649503 PMid:29791947

7. Giroud $M$, Jacquin $A$, Béjot $Y$. The worldwide landscape of stroke in the $21^{\text {st }}$ century. Lancet. 2014;383(9913):195-7. https:// doi.org/10.1016/s0140-6736(13)62077-2

PMid:24449941 
8. Kim AS, Cahill E, Cheng NT. Global stroke belt geographic variation in stroke burden worldwide. Stroke. 2015;46(12):356470. https://doi.org/10.1161/STROKEAHA.115.008226 PMid:26486867

9. Jantasri S, Tiamkao S, Sawanyawisuth K. A 2-point difference of NIHSS as a predictor of acute ischemic stroke outcome at 3 months after thrombolytic therapy. Clin Neurol Neurosurg. 2020;198:106206. https://doi.org/10.1016/j. clineuro.2020.106206

PMid:32932029

10. Kijpaisalratana N, Chutinet A, Akarathanawat W, Vongvasinkul $P$, Suwanwela NC. Outcomes of thrombolytic therapy in acute ischemic stroke: Mothership, drip-and-ship, and ship-and-drip paradigms. BMC Neurol. 2020;20(1):45. https://doi.org/10.1186/ s12883-020-1631-9 PMid:32013906

11. Man S, Xian Y, Holmes DN, Matsouaka RA, Saver JL, Smith EE, Bhatt DL, et al. Association between thrombolytic door-to-needle time and 1-year mortality and readmission in patients with acute ischemic stroke. JAMA. 2020;323(21):2170-84. https://doi. org/10.1001/jama.2020.5697

PMid:32484532

12. Sriratanaban J, Ngamkiatpaisarn $\mathrm{S}$, Charoenmukayananta $\mathrm{S}$. Association between hospital accreditation and outcomes: The analysis of inhospital mortality from the national claims data of the universal coverage scheme in Thailand. Qual Manag Health Care. 2020;29(3):150-7. https://doi.org/10.1097/ QMH.0000000000000256 PMid:32590490

13. Schlemm L, Endres M, Werring DJ, Nolte $\mathrm{CH}$. Benefit of intravenous thrombolysis in acute ischemic stroke patients with high cerebral microbleed burden. Stroke. 2020;51(1):232-9. https://doi.org/10.1161/strokeaha.119.027633 PMid:31739772

14. Barlas RS, Clark AB, Bettencourt-Silva JH, Sawanyawisuth K, Kongbunkiat $\mathrm{K}$, Kasemsap $\mathrm{N}$, et al. Pneumonia and risk of serious adverse outcomes in hospitalized strokes in Thailand. J Stroke Cerebrovasc Dis. 2019;28(6):1448-54. https://doi. org/10.1016/j.jstrokecerebrovasdis.2019.03.024 PMid:30956056

15. Reid KA, Barlasm RS, Mamas MA, Clark AB, Kwok CS, Wong $C W$, et al. Infective endocarditis is associated with worse outcomes in stroke: A Thailand national database study. Int J Clin Pract. 2020;74(11):e13614. https://doi.org/10.1111/ijcp.13614

\section{PMid:32688452}

16. Szlachetka WA, Pana TA, Tiamkao S, Clark AB, Kongbunkiat $K$ Sawanyawisuth K, et al. Impact of diabetes on complications, long term mortality and recurrence in 608, 890 hospitalised patients with stroke. Glob Heart. 2020;15(1):2. https://doi. org/10.5334/gh.364

PMid: 32489775

17. Pana TA, Wood AD, Perdomo-Lampignano JA, Tiamkao $S$ Clark $A B$, Kongbunkiat $\mathrm{K}$, et al. Impact of heart failure on stroke mortality and recurrence. Heart Asia. 2019;11(1):e011139. https://doi.org/10.1136/heartasia-2018-011139 PMid:31244914

18. Tung IM, Barlas RS, Vart $P$, Bettencourt-Silva JH, Clark AB Sawanyawisuth $\mathrm{K}$, etal. Association of chronic kidney disease with outcomes in acute stroke. Acta Neurol Belg. 2021;121(5):12416. https://doi.org/10.1007/s13760-020-01416-0 PMid:32661738

19. Cumming K, Tiamkao S, Kongbunkiat K, Clark AB, BettencourtSilva JH, Sawanyawisuth K, et al. Impact of HIV on inpatient mortality and complications in stroke in Thailand: A national database study. Epidemiol Infect. 2017;145(6):1285-91. https:// doi.org/10.1017/S095026881600340X

PMid:28137330

20. Wood AD, Mannu GS, Clark AB, Tiamkao S, Kongbunkiat $K$ Bettencourt-Silva $\mathrm{JH}$, et al. Rheumatic mitral valve disease is associated with worse outcomes in stroke: A Thailand national database study. Stroke. 2016;47(11):2695-701. https://doi. org/10.1161/strokeaha.116.014512 PMid:27703088

21. di Poggio MB, Finocchi C, Brizzo F, Altomonte F, Bovis F, Mavilio $\mathrm{N}$, et al. Management of acute ischemic stroke, thrombolysis rate, and predictor of clinical outcome. Neurol Sci. 2019;40(2):319-26. https://doi.org/10.1007/s10072-018-3644-3 PMid:30430315

22. Pinhoa J, Costa AS, Araújo JM, Amorim JM, Ferreir C Intracerebral hemorrhage outcome: A comprehensive update. J Neurol Sci. 2019;398:54-66. https://doi.org/10.1016/j. jns.2019.01.013

PMid:30682522

23. Garg R, Biller J. Recent advances in spontaneous intracerebral hemorrhage. F1000Res. 2019;8:F1000. https://doi. org/10.12688/f1000research.16357.1

PMid:30906532 